\title{
CYCLIC NEVANLINNA CLASS FUNCTIONS IN BERGMAN SPACES
}

\author{
PAUL BOURDON
}

\begin{abstract}
Let $f$ be a function which is in both the Bergman space $A^{p}$ $(p \geq 1)$ and the Nevanlinna class $N$. We show that if $f$ is expressed as the quotient of $H^{\infty}$ functions, then the inner part of its denominator is cyclic. As a corollary, we obtain that $f$ is cyclic if and only if the inner part of its numerator is cyclic. These results extend those of Berman, Brown, and Cohn [2]. Using more difficult methods, they have obtained them for the case $f \in A^{2} \cap N$. Finally, we show that the condition $|f(z)| \geq \delta(1-|z|)^{c}(z \in D ; \delta, c$ positive constants) is sufficient for cyclicity for $f \in A^{p} \cap N$, which answers a question of Aharonov, Shapiro, and Shields [1] .
\end{abstract}

1. Introduction. For $1 \leq p<\infty$, let $A^{p}$ denote the Bergman space consisting of those functions $f$ holomorphic on the open unit disk $D$ satisfying $\|f\|_{p}<\infty$, where

$$
\|f\|_{p}^{p}=\frac{1}{\pi} \int_{0}^{2 \pi} \int_{0}^{1}\left|f\left(r e^{i \theta}\right)\right|^{p} r d r d \theta
$$

The norm \|\|$_{p}$ makes $A^{p}$ into a Banach space in which the polynomials are dense.

For $f \in A^{p}$ define $[f]$ by $[f]=A^{p}$-closure of $\{p f: p$ is a polynomial $\}$. We say that a function $f \in A^{p}$ is cyclic in $A^{p}$ provided it is cyclic for the forward shift operator $T_{z}$ on $A^{p}$, where $T_{z}$ is defined by $\left(T_{z} f\right)(z)=z f(z)$. Thus $f$ is cyclic in $A^{p}$ if $[f]=A^{p}$. It is not difficult to verify that $[f]=A^{p}$-closure of $\left\{h f: h \in H^{\infty}(D)\right\}$ and that the following are equivalent.

(a) $f$ is cyclic in $A^{p}$.

(b) There is a sequence $\left\{p_{n}\right\}$ of polynomials such that $\left\|p_{n} f-1\right\|_{p} \rightarrow 0$.

(c) There is a sequence $\left\{h_{n}\right\}$ of $H^{\infty}$ functions such that $\left\|h_{n} f-1\right\|_{p} \rightarrow 0$.

Let $S \mu$ denote the singular inner function induced by $\mu$; that is,

$$
S \mu(z)=\exp \left(-\int_{T} \frac{\omega+z}{\omega-z} d \mu(\omega)\right) .
$$

Here, of course, $\mu$ is a positive finite Borel measure on the unit circle $T$ singular with respect to Lebesgue measure. The Nevanlinna class $N$ consists of those functions $f$ holomorphic on $D$ which satisfy

$$
\sup _{r<1} \int_{0}^{2 \pi} \log ^{+}\left|f\left(r e^{i \theta}\right)\right| d \theta<\infty .
$$

If $f \in N$, then $f$ factors as $B \gamma S \nu / \phi S \mu$, where $B$ is a Blaschke product, $\gamma$ and $\phi$ are outer functions in $H^{\infty}$, and where $\nu$ and $\mu$ are mutually singular measures $(\nu \perp \mu)$ [3, p. 25].

Received by the editors March 21, 1984 and, in revised form, May 1, 1984.

1980 Mathematics Subject Classification. Primary 47B37; Secondary 30D50.

(C) 1985 American Mathematical Society $0002-9939 / 85 \$ 1.00+\$ .25$ per page 
We now establish two easy propositions for future reference.

PROPOSITION 1. If $\gamma \in H^{\infty}$ is outer, then $\gamma$ is cyclic in $A^{p}$.

ProOF. That $\gamma$ is cyclic in $H^{p}$ follows from Beurling's Theorem [3, p. 114]; however, convergence in $H^{p}$ implies convergence in $A^{p}$.

Proposition 2. $f \in H^{\infty}$ is cyclic in $A^{p}$ if and only if its inner part is cyclic in $A^{p}$.

PROOF. It is clear that cyclic vectors in $A^{p}$ must be nonzero; hence, $f$ factors as $\gamma S \mu$ where $\gamma$ is outer and in $H^{\infty}$. We show that $[f]=[S \mu]$. Since $f$ is an $H^{\infty}$ multiple of its inner part $S \mu,[S \mu] \supset[f]$. The outer function $\gamma$ is cyclic, so there is a sequence $\left\{p_{n}\right\}$ of polynomials such that $\left\|p_{n} \gamma-1\right\|_{p} \rightarrow 0$. Now, making use of the fact that $\|h g\|_{p} \leq\|h\|_{\infty}\|g\|_{p}$ for $g \in A^{p}$ and $h \in H^{\infty}$, we find $\left\|p_{n} \gamma S \mu-S \mu\right\|_{p} \leq\left\|p_{n} \gamma-1\right\|_{p}$ so that $S \mu \in[f]$. It follows that $[S \mu]=[f]$.

We have the following characterization of cyclic inner functions in $A^{p}$.

THEOREM. S $\mu$ is cyclic in $A^{p}$ if and only if $\mu$ places no mass on any Carleson set.

Necessity was proved by H. S. Shapiro [8], sufficiency by B. Korenblum $[\mathbf{4}, \mathbf{6}]$ and (independently) J. Roberts $[\mathbf{7}, \mathbf{9}]$. Carleson sets are certain compact sets of measure zero in $T$. Specifically, a compact set $K \subset T$ is Carleson if $\int_{T} \log \rho_{K}(\omega) d m(\omega)>$ $-\infty$, where $\rho_{K}(\omega)=\operatorname{dist}(\omega, K)$ and $m$ is Lebesgue measure on $T$.

2. Results. We postpone the proof of the following theorem until after presenting some of its consequences.

THEOREM 1. If $f \in A^{p} \cap N$, then $f=B \gamma S \nu / \phi S \mu$ where $S \mu$ is cyclic in $A^{p}$.

Corollary 1. $f \in A^{p} \cap N$ is cyclic in $A^{p}$ if and only if $f=\gamma S \nu / \phi S \mu$ where $S \nu$ is cyclic.

Proof. If $S \nu$ is cyclic then $\gamma S \nu$ is cyclic (Proposition 2). But $\gamma S \nu=(\phi S \mu) f$ so that $[f]$ contains the cyclic vector $\gamma S \nu$. Thus $f$ is cyclic.

Conversely, suppose $f$ is cyclic. Since cyclic vectors are nonzero, $f$ factors as $\gamma S \nu / \phi S \mu$. Let $\left\{p_{n}\right\}$ be a sequence of polynomials such that $\left\|p_{n} f-1\right\|_{p} \rightarrow 0$. Then

$$
\begin{aligned}
\left\|p_{n} \gamma S \nu-\phi S \mu\right\|_{p} & =\left\|\phi S \mu\left(p_{n} \frac{\gamma S \nu}{\phi S \mu}-1\right)\right\|_{p} \\
& \leq\|\phi\|_{\infty}\left\|p_{n} f-1\right\|_{p} ;
\end{aligned}
$$

consequently, $\phi S \mu \in[\gamma S \nu]$. However, $\phi S \mu$ is cyclic by Theorem 1 ; thus $S \nu$ is cyclic.

Corollary 2. If $f \in A^{p} \cap N$ and if $1 / f \in A^{p}$, then $f$ is cyclic in $A^{p}$.

ProOF. Let $f=\gamma S \nu / \phi S \mu$. $S \nu$ is cyclic since $1 / f \in A^{p}$; therefore, $f$ is cyclic by Corollary 1 .

REMARK. Using different methods, Berman, Brown, and Cohn [2] have obtained Theorem 1 as well as Corollaries 1 and 2 for the case $f \in A^{2} \cap N$. 
THEOREM 2. If $f \in A^{p} \cap N$ satisfies $|f(z)| \geq \delta(1-|z|)^{c}$ for $z \in D(\delta$, c positive constants) then $f$ is cyclic in $A^{p}$.

Proof. Again, let $f=\gamma S \nu / \phi S \mu$. Note that $f$ has an analytic $n$th root since it is nonzero on $D$. Choose $n$ large enough so that $c / n \leq 1 / 2 p$. It is easy to check that both $f^{1 / n}$ and $f^{-1 / n}$ are in $A^{p}$. We see that $f^{1 / n}$ is cyclic by Corollary 2 , and consequently $\nu / n$ places no mass on any Carleson set (Corollary 1 ). Therefore, $\nu$ places no mass on any Carleson set and $S \nu$ is cyclic. Now, $f$ is cyclic by Corollary 1.

3. Proof of Theorem 1. An inner function $q$ is said to be $B$-inner if it divides every inner function in $[q]$. The following theorem was established independently by James Roberts [7] and Pat Ahern [9].

THEOREM 3. $S \mu=S \mu_{b} S \mu_{c}$ where $S \mu_{b}$ is B-inner and $S \mu_{c}$ is cyclic.

Ahern has shown that the $B$-inner, cyclic factorization in the Bergman spaces is a corollary of the Shapiro-Korenblum-Roberts characterization of cyclic inner functions, while Roberts has proved the existence of such a factorization in a somewhat more general setting without using the cyclic inner function characterization. Both Roberts and Ahern have shown that in the Bergman spaces, $S \mu$ is $B$-inner if and only if $\mu$ is concentrated on a countable union of Carleson sets.

ProOF OF THEOREM 1. Recall $f=B \gamma S \nu / \phi S \mu$ where $\nu \perp \mu$. Let $\left\{p_{n}\right\}$ be a sequence of polynomials such that $\left\|p_{n}-f\right\|_{p} \rightarrow 0$. Then

$$
\begin{aligned}
\left\|p_{n} \phi S \mu-B \gamma S \nu\right\|_{p} & =\left\|\phi S \mu\left(p_{n}-B \gamma S \nu / \phi S \mu\right)\right\|_{p} \\
& \leq\|\phi\|_{\infty}\left\|p_{n}-f\right\|_{p} ;
\end{aligned}
$$

consequently, $B \gamma S \nu \in[\phi S \mu]$. But $\gamma$ is outer and in $H^{\infty}$; therefore, we have, just as in the proof of Proposition 2, $B S \nu \in[B \gamma S \nu]$. Hence, $B S \nu \in[\phi S \mu]$. Thus there is a sequence $\left\{q_{n}\right\}$ of polynomials such that $\left\|q_{n} \phi S \mu-B S \nu\right\|_{p} \rightarrow 0$.

We have $\left\|\left(q_{n} \phi S \mu_{c}\right) S \mu_{b}-B S \nu\right\|_{p} \rightarrow 0$ so that in fact, $B S \nu \in\left[S \mu_{b}\right]$. Now, by the definition of $B$-inner, there exists an inner function $q$ such that $B S \nu=q S \mu_{b}$. It follows that $S \mu_{b} \mid S \nu$; however, $\mu_{b} \perp \nu$ and hence $S \mu_{b}=1$. Thus $S \mu=S \mu_{c}$ is cyclic.

REMARKS. 1. Corollary 1 may be proved directly using an argument similar to the one used in the proof of Theorem 1.

2. Since Shapiro-Korenblum-Roberts characterization of cyclic inner functions remains valid in the Bergman spaces $A_{\alpha}^{p}$ with $\alpha>-1$ and $0<p<\infty$, our results extend readily to these spaces. Here the subscript $\alpha$ indicates that the Bergman norm is induced by the weighted measure $(1-r)^{\alpha} r d r d \theta$.

ACKNOWLEDGMENT. I would like to thank my advisor, J. A. Cima, Warren Wogen, and the referee for helpful suggestions.

\section{REFERENCES}

1. D. Aharonov, H. S. Shapiro and A. L. Shields, Weakly invertible elements in the space of square summable holomorphic functions, J. London Math. Soc. (2) 9 (1974), 183-192.

2. R. Berman, L. Brown and W. Cohn, Cyclic vectors of bounded characteristic in Bergman spaces, preprint.

3. P. Duren, Theory of $H^{p}$ spaces, Academic Press, New York, 1970. 
4. B. Korenblum, An extension of the Nevanlinna theory, Acta Math. 135 (1975), 187219.

5. __ A Beurling-type theorem, Acta Math. 138 (1977), 265-293.

6. __ Cyclic elements in some spaces of analytic functions, Bull. Amer. Math. Soc. (N.S.) 5 (1981), 317-318.

7. J. Roberts, Cyclic inner functions in the Bergman spaces and weak outer functions in $H^{p}, 0<p<1$. Illinois J. Math. (to appear).

8. H. S. Shapiro, Some remarks on weighted polynomial approximations by holomorphic functions, Math. U.S.S.R. Sbornik 2 (1967), 285-294.

9. J. Shapiro Cyclic inner functions in Bergman spaces, unpublished seminar notes on the results of H. S. Shapior and J. Roberts (1980).

Department of Mathematics, University of North Carolina at Chapel Hill. Chapel hill, North Carolina 27514 